Résumés des conférences et travaux

\title{
Sociolinguistique diachronique romane
}

\section{Michel Banniard}

\section{OpenEdition \\ Journals}

Édition électronique

URL : https://journals.openedition.org/ashp/2427

DOI : $10.4000 /$ ashp. 2427

ISSN : 1969-6310

Éditeur

Publications de l'École Pratique des Hautes Études

\section{Édition imprimée}

Date de publication : 1 septembre 2018

Pagination : 190-193

ISSN : 0766-0677

Référence électronique

Michel Banniard, "Sociolinguistique diachronique romane ", Annuaire de l'École pratique des hautes études (EPHE), Section des sciences historiques et philologiques [En ligne], 149 | 2018, mis en ligne le 05 juillet 2018, consulté le 03 août 2021. URL : http://journals.openedition.org/ashp/2427 ; DOI : https:// doi.org/10.4000/ashp.2427 


\title{
SOCIOLINGUISTIQUE DIACHRONIQUE ROMANE
}

\author{
Directeur d'études : M. Michel BANNIARD
}

Programme de l'année 2016-2017 : I. Vitae mérovingiennes et compromis communicationnels $\left(V I^{e}-V I I I^{e} s.\right)$. - II. Chartae d'Italie et genèse de la langue italienne (VIII ${ }^{e}-I X^{e}$ s. $)$. - III. Gesta Karoli ad Carcassonam et reconquête médiatique (XII $\left.{ }^{e}-X I I I^{e} s.\right)$. - IV. Peire Vidal. Syntaxe complexe et langue des élites (ca 1180-1200).

Le séminaire s'est inscrit dans la continuité des séances de l'année précédente avec un certain nombre de modifications selon les découvertes récentes ou les demandes de certains participants. La publication d'une collection de sermons a servi de base de réflexion initiale sur les fluctuations considérables du niveau de la langue écrite, précisément à la fin de la période de transition du LPT2 (latin parlé tardif de phase 2, $\mathrm{VI}^{\mathrm{e}}-\mathrm{VII}^{\mathrm{e}} \mathrm{s}$.), au PR (protoroman, VIII ${ }^{\mathrm{e}}$ s.) : Stephen Pelle, «An Edition of an Unstudied Early Carolingian Sermon Collection », The Journal of Medieval Latin, t. 23, 2013, p. 87-160. Cette collection de 19 sermons, datable des années 800 , est restée intacte à Saint-Gall. On est revenu sur les intéressants commentaires linguistiques de l'éditeur (p. 93-95) pour d'abord approuver ses observations sur les destinataires de ces petits sermons, « ... all suggest that the primary audience for these sermons was the common, uneducated public ». Puis pour corriger sensiblement son commentaire, encore fondé sur une méthode « déviationniste » par rapport aux normes du latin classique et sur le modèle dualiste du « latin vulgaire ». On a donc traduit et épluché la page 106 (le péché originel au jardin d'Eden) pour montrer que sur l'échelle en 5 niveaux élaborée pour rendre compte de la place de ce «latin » en diachronie longue, la langue employée tout en respectant une graphie fidèlement latinisante et en esquivant les romanismes les plus marqués, trie et organise, sa morphologie, sa syntaxe, son phrasé et son lexique qui relèvent du niveau 4 , voire 5 parfois, autrement dit du protoroman latiniforme. On a complété le travail en lisant à haute voix quelques passages en oralité romane, calquée sur ce que nous savons du protofrançais. Ce fut alors l'occasion de comparer ce niveau de langue avec un certain nombre de passages de même niveau dans les diplômes, chartes et documents carolingiens « pragmatiques ».

Le thème « mérovingien » a été abordé en reprenant le dossier de la Vita Praeiecti episcopi arvernensis, ouvert l'année précédente à la requête d'une historienne médiéviste (Isabelle Réal), dans l'édition des $M G H$ (SRM, t. V, p. 212-245, mais en reprenant le texte dans son intégralité, à commencer par la présentation des manuscrits établie par B. Krusch (p. 219-223), le plus ancien remontant aux alentours de l'an mil (codex Rothomagensis). C'est lui que suit l'éditeur qui déplore l'incorrection extrême de sa langue : moment de reposer le problème en termes modernes, à la lumière des nouveaux travaux dont fait l'objet la latinité hagiographique mérovingienne par une remise en chantier précisément de sa tradition manuscrite (la numérisation permet désormais d'ouvrir commodément ces domaines) et l'appropriation des approches modernes de la linguistique diachronique. L'exercice pratique s'est d'abord appuyé sur la lecture et la traduction de l'épitaphe du prédécesseur de Priest, saint Genès 
(p. 213), qui réclamait au moins une correction, ARCA pour ARVA, et présentait sinon une langue de bonne tenue malgré quelques flottements graphiques (PLEVI pour PLEBI, EDE pour AEDEM, FABRECA pour FABRICA...), et une terminologie conforme aux conclusions de Martin Heinzelmann sur la permanence d'une mentalité aristocratique sénatoriale.

Nous avons alors laissé cette figure du $\mathrm{VII}^{\mathrm{e}}$ siècle pour remonter le temps à la demande d'un étudiant de master 2 en histoire médiévale (Yoan Solirenne) qui, travaillant sur les sceaux, s'était trouvé confronté à une source importante, mais difficile, la lettre LXXXVII, écrite vers 509 par Avit, alors évêque de Vienne ( $M G H, A A$, t. VI, Pars Posterior, p. 96-97). Avit y répond à son frère Apollinaris, lui-même évêque de Valence, qui lui a promis de commander pour lui un anneau sigillaire épiscopal. La lettre constitue un chef-d'œuvre de prose savante, où le récipiendaire décrit par le menu l'objet qu'il souhaite recevoir, en pratiquant une ekphrasis magistrale dans un latin lui-même au-dessus du niveau 1, autrement dit passablement compliqué. Après une présentation du dossier historique et archéologique par l'étudiant, on est passé à la lecture et à la traduction du texte, en s'appuyant parfois sur quelques variantes manuscrites pour en éclairer le sens (magdali olla en apparat, plutôt que magdaliola illa édité, mais incompréhensible). On a terminé par trois apports : un dessin de l'objet tracé au tableau par un des auditeurs habile à cet art (Pierre Marillaud); une analyse rapide du maniement des cas obliques (hyperconservateur); par des réflexions sur les raisons de cet attachement à une langue si sophistiquée pour en déduire que, tout évêques qu'ils étaient devenus, ces puissants avaient gardé profondément en et entre eux les marques de la connivence sociale "païenne » du temps de l'Empire, passant par l'étiquette, la majesté, la fulgurance langagière (comme Sidoine Apollinaire). Tout ceci suppose au moins au début $\mathrm{du} \mathrm{VI}^{\mathrm{e}}$ siècle un tissu culturel de haut niveau soigneusement entretenu au sein des lignages aristocratiques - du moins dans les régions considérées. Il y aura lieu de vérifier dans quelle mesure les héritiers du $\mathrm{VII}^{\mathrm{e}}$ siècle (Desiderius, Praeiectus...) conservent cette structure mentale et ce savoir.

Traduction (de travail...).

L'évêque Avit à l'évêque Apollinaire.

J'ai pleine confiance et je ne doute pas que sous l'effet d'un cadeau divin une joie commune ne soit annoncée à notre commune présence. Car, notice que je suppose vous être déjà parvenue, même ceux dont on disait qu'ils ravageaient la frontière, sont rentrés chez eux. C'est pourquoi je vous ai adressé par sollicitude cette lettre afin que vous jugiez ensuite de l'amélioration de la sécurité pour chacun : à moins qu'un repli en liberté vers la cité ne vous ait arraché à l'habitude ou plutôt à l'amour du siège. De ce fait, j'attends que vous ordonniez l'envoi de cette jarre avec une note de recette.

Assez de familiarités. À présent, je vais donner des précisions avec un peu plus d'allégresse sur le reste, puisque c'est l'instant. Donc, «l'outil à signer», que votre piété a daigné moins promettre qu'offrir, sera réalisé à mon gré de la façon suivante.

$\grave{A}$ un anneau de fer très fin, qui se fermera avec deux minidauphins courant quasiment l'un vers l'autre, on insèrera la forme mobile d'un sceau à double face, monté sur un axe géminé qui selon les convenances se transformera en alternance aux regards de ceux qui examinent les documents, soit privés, soit publics, en montrant alternativement le front soit d'une gemme verte, soit d'un ambre pâle. 
Et pas en tous cas celui d'un ambre tel que celui qui tout récemment, lorsque je l'ai moimême puisé, gisait sans éclat dans la blancheur sainte d'une main non polluée, et dans lequel on aurait cru se trouver un mélange plutôt dégradé que parfait d'un or pas encore purifié par le four : ni même de l'ambre que le roi des Gètes avait adressé tout récemment comme présage de la ruine qui allait suivre pour la monnaie publique, parce qu'il était manifestement impur. Mais que sa couleur soit d'un type tel que la délicatesse de sa texture démontre avec une régularité modeste qu'il tire sa rougeur de l'or, sa blancheur de l'argent, sa brillance aveuglante de tout le reste, en un excès d'art et en même temps d'extrême mesure.

Si tu demandes ce qu'il faut graver sur le sceau : que le symbole de mon monogramme soit enroulé à l'indication de mon nom le long du cercle.

En plus, les queues des dauphins, dont nous avons décrit plus haut les têtes, occuperont le milieu de l'anneau sur la partie où il s'approchera de la fermeture de l'arc. Dans ces queues sera scellée une pierre précieuse recherchée à cet effet, c'est-à-dire sculptée en longueur avec des extrémités pointues.

Et voilà, tu as à peu près une image du dogme à respecter. Malgré tout, je n'enserre pas l'ampleur de ton raffinement dans le modèle indiqué au point qu'il ne te soit pas loisible d'y ajouter ce que bon te semblera. Ah oui, il sera vraiment permis à ton talent lancé au galop, de dépasser les limites de la requête suscrite.

Au terme de ma lettre, mon propos bourbeux réclame, pour le travail de l'argile sans lequel on ne peut de toutes façons arriver à créer, que vous guidiez l'artiste potier, en le contrôlant pas à pas, pour qu'il mette en place pour nous selon quelles mesures pourra être disposé le foyer à trous et dans quelle taille d'une fosse de glèbe extraite d'un sol entouré des pieds sordides des animaux, l'agglomérat de boue à cuire pourra être liquéfié.

Le thème des Gesta Karoli, après une série de lectures suivant de près le texte latin et le texte occitan, a été alors clôturé. D'abord en revenant sur l'inexactitude du nom usuel «le roman de Philomène », peu approprié pour désigner le texte original, qui était bien latin, la version occitane étant clairement une traduction faite sans doute immédiatement après, contrairement à la légende héritée du XIX ${ }^{\mathrm{e}}$ siècle, elle-même dépendant d'une compréhension erronée de la préface latine de l'œuvre et du sens de l'apparition du fameux Philomène, premier supposé rédacteur carolingien des Gesta (mythiquement compris comme un poète épique «provençal »). La comparaison précise du latin et de l'occitan confirme (c'est précisément l'avis du dernier éditeur Heitzmann) sans aucun doute que le latin est bien la langue source, que le traducteur suit adroitement mais en gardant le phrasé original (et en ajoutant quelques détails relevant de la mentalité « laïque »). On a terminé par une mise au point sur les caractères stylistiques et langagiers de ce latin : très linéaire et souple, il est en fait matricé tant pour une lecture à haute voix " latinisante » à l'usage d'élites dont le niveau de latinité était moyen; soit bien plutôt afin de pouvoir le transferre directement à haute voix en occitan (les adaptations étaient suffisamment limitées pour pouvoir le faire en direct). Mais il y a lieu de supposer que ces mêmes destinataires (l'aristocratie laïque occitanophone) ont rapidement exigé de pouvoir lire directement ce récit capital pour leurs intérêts. D'où la traduction. La session été close par la lecture, la traduction et le commentaire de la charte originale (et authentique) de donation de 779 (éditée tant dans le recueil procuré par E. Magnou-Nortier que dans les Chartae latinae antiquiores). 
Le séminaire s'est alors penché sur proposition d'un doctorant en histoire médiévale (Denis Mirouse) sur la Convention passée entre Pierre évêque et Roger son neveu (vers 1030-vers 1050). Le doctorant a apporté des reproductions des originaux, donné leur transcription et procédé à l'interprétation historique (enrichie d'une cartographie des lieux de la transaction) de cette convention compliquée, en présence et avec l'appui de sa directrice (H. Debax). Le directeur a de son côté commencé par reprendre la typologie des niveaux de langue dans des documents latiniformes antérieurs pour l'appliquer ensuite au texte étudié. On a ensuite épluché la succession des énoncés en les étiquetant, ce qui a permis de vérifier que l'occitan juridique parlé par les protagonistes a matricé la quasi-totalité des énoncés, à peine toilettés par brève séquence de quelques latinismes légalistes. On a relevé que c'est la seule graphie qui crée par moment l'illusion latine en ce qu'elle masque la phonie réelle, qui apparaît directement dans la lecture soit en voix intérieure, soit à haute voix :

... ipsa civitate de Carcassona cum ipso comitatu de Carcassense et cum ipsos alodes comitales et cum ipsa honore de ipso comitatu mittit Petrus episcopus ad una parte, excepto ipso episcopatu de Carcassona...

On voit comment la graphie latiniforme contribue à établir la scripta occitane proprement dite, qui apparaît finalement de plus en plus à mesure que le rédacteur et les protagonistes développent le détail de leurs arrangements :

Et ipso comitivo de ipso Bancel qui es infre Lural e Casal marcu tro ad Aregia, sia d'aquel qui tenrra Fox... E de Savardo tro a iusta red da Reiga enlà, e de iusta red tro en Bolbestres, sia d'aquel lo comtius...

Si le découpage des mots est stable dans la graphie latiniforme, il flotte un peu en graphie occitane, ce qui requiert des corrections : da quel $>$ d'aquel; en la $>$ enlà. Mais tout ce petit monde paraît langagièrement très à son aise, et le rédacteur pratique allègrement non le code switching (en fait, il ne change pas de langue), mais le graphic switching. 